\title{
Réflexions sur le Système Monétaire Européen *
}

\author{
par Robert Triffin **
}

\begin{abstract}
Afin de bien me faire comprendre, j'essaierai de grouper mes remarques sous trois parties, sous trois titres essentiels.

Le premier : l'origine et les motivations de la création du système monétaire européen, le second: ses traits essentiels aujourd'hui et le troisième: ses perspectives d'avenir.
\end{abstract}

\section{I}

Tout d'abord, origine et motivations. Je crois que ce qui est à l'origine de la création du système monétaire européen, c'est un accord presque unanime sur la nécessité de remédier enfin au chaos hérité de deux facteurs. Primo, l'effondrement évident du système de Bretton Woods, effondrement consacré légalement en août 1971 mais en fait bien antérieur ; et secondo, l'abdication, temporaire je l'espère, de dix ans d'efforts de réforme monétaire internationale qui semblaient en 1973 prêts d'aboutir à un consensus très général.

Je susciterai, j'espère, une discussion animée en disant que ce qui en est résulté finalement, le second amendement aux statuts du Fonds Monétaire International, n'est pour moi qu'une sinistre plaisanterie. J'expliquerai pourquoi tout à l'heure si le temps me le permet.

Je crois que la création du système monétaire européen vise avant tout à réduire la dépendance excessive du système monétaire de l'Europe vis-à-vis du dollar utilisé comme monnaie parallèle de règlement, d'accumulation de réserves, etc., tant par les entités privées que par les banques centrales et les gouvernements. Ce système s'est en fait révélé depuis quinze ou vingt ans un engin de transmission d'une inflation mondiale bien antérieure à la crise du pétrole. Il est évidemment facile de blâmer l'inflation actuelle sur la crise du pétrole, mais n'oubliez pas que la suspension de la convertibilité en or du dollar est intervenue en 1971, c'est-à-dire plus de deux ans avant l'explosion des prix du pétrole et que l'inflation mondiale mesurée par les prix mondiaux d'importation et d'exportation exprimés en dollars atteignait déjà un rythme annuel de $30 \%$ au cours des douze mois qui ont précédé l'explosion des prix du pétrole.

Ce qu'on essaie de faire, c'est d'isoler dans la mesure du possible les économies européennes des chocs provoqués par des réactions nationales divergentes aux crises répétées du dollar, réactions nationales divergentes de pays à pays et non seulement

* Conférence prononcée le 9 juillet 1979 à Madrid, à l'occasion de l'Assemblée Annuelle des membres de l'"Association de Genève ».

** Professeur aux Universités de Yale et Louvain. 
de pays à pays, mais à l'intérieur même du même pays. Prenez comme exemple l'Allemagne ou la Suisse à des moments différents des dernières années.

Ces deux réactions aux crises répétées du dollar sont les suivantes : primo, intervenir pour empêcher une baisse du dollar afin de protéger les producteurs nationaux contre une concurrence jugée excessive, à tort ou à raison, des producteurs américains chez soi et dans le monde, fût-ce au prix d'un financement inflationniste. C'est là la réaction lorsque dominent surtout les craintes de récession et de chômage. Secondo, refuser ce financement et laisser tomber le dollar, fût-ce au prix de récession et de chômage, afin de freiner l'inflation dans son propre pays. Et vous avez vu l'Allemagne, vous avez vu la Suisse, passer tous les trois ou tous les quatre mois d'une réaction à l'autre et certains pays réagissant davantage dans un sens et d'autres dans l'autre. Je crois que tout cela affecte évidemment les relations entre pays européens et qu'il y a moyen de faire mieux que ce que l'on a fait à cet égard.

Je mentionnerai incidemment un autre argument que je vois brandir constamment dans la presse à propos du désir que l'on aurait de laisser tomber le dollar. C'est l'argument que, comme le pétrole est payé en dollar, la chute de la valeur du dollar en termes de certaines autres monnaies plus fortes, réduira pour ces pays le coût de leurs importations de pétrole. Je crois que c'est là un argument extrêmement dangereux.

Je reviens d'Abbou d'Abi et du Koweit et je vous assure que ces gens ne sont pas des imbéciles. Ils se rendent parfaitement compte de cet argument, mais se disent que dans ce cas-là ils augmenteront davantage les prix en dollars ou changeront d'unité de compte pour leurs exportations de pétrole.

J'en viens à un second point.

\section{II}

Quels sont les traits essentiels du système monétaire européen? J'aimerais tout d'abord dissiper une erreur qui, au point de départ, avait rendu extrêmement sceptiques une série d'économistes et notamment les économistes américains. Le système monétaire européen, enfin mis en place en mars dernier, n'est pas encore l'Union monétaire européenne, ni même la stabilité immédiate et irrévocables des taux de change entre monnaies européennes. Pour arriver à cela, il faudra un saut qualitatif et politique sur lequel l'accord n'est hélas pas encore totalement fait en dépit des proclamations répétées de nos chefs d'Etat et de gouvernement dans leurs réunions au sommet. Mais c'est un premier pas, tout au moins vers cette union monétaire, que l'on peut espérer reprendre un jour, un pas indispensable, un pas séminal, un pas qui, s'il réussit, déclenchera lui-même d'autres réactions logiques et internes ne demandant pas chaque fois l'intervention de décisions répétées des gouvernements.

Pour être bref, je me limiterai à quatre points essentiels du nouveau système monétaire européen.

1) Le premier trait essentiel, c'est la définition d'une unité monétaire commune, l'écu, c'est-à-dire un panier de monnaie qui reflète la valeur moyenne pondérée des différentes monnaies des pays participants, mais vis-à-vis duquel les monnaies nationales de l'Europe continueront pendant un certain temps encore à fluctuer ou à être réajustées de temps en temps. Ceci est essentiel à divers égards. Tout d'abord, et me 
référant de nouveau à ce que j'appelais tout à l'heure une sinistre plaisanterie, il est véritablement invraisemblable que, dans les statuts actuels du Fonds Monétaire International, il n'y ait même plus d'instrument de mesure commune. Il y a encore un chapitre qui parle des obligations concernant les régimes de change et ce chapitre, très long et très compliqué, peut se résumer en deux mots. C'est que chaque pays peut adopter le régime de change qu'il voudra, sauf un seul, qui était le seul qui était légal jusque-là, c'est-à-dire de définir sa monnaie en or. Cela c'est interdit mais à part ça, on peut définir sa monnaie en dollars, en francs français, en marks allemands ou dans un panier de monnaies différent d'un pays à l'autre. Il n'y a plus de mètre, il n'y a plus d'étalon commun, il n'y a plus de numéraire.

L'écu offre un nouveau numéraire qui est l'écu lui-même vis-à-vis duquel chaque pays définira la valeur de sa monnaie, son taux de change, et réajustera éventuellement ces taux de change de la manière la plus logique. Il était parfaitement logique autrefois de définir toutes les monnaies en or, mais pratiquement en dollars, lorsque le dollar était la monnaie universellement acceptée et convertible en or. Mais ce ne l'est plus parce que le dollar lui-même devient une pure monnaie de papier fluctuant de jour en jour, vers le haut et hélas vers le bas.

A ce moment-là, l'intérêt de chaque pays est d'essayer de définir la stabilité de sa monnaie, les réajustements de sa monnaie, vis-à-vis des pays avec lesquels il fait la plus grande partie de son commerce. C'est ainsi que la plupart des pays ont adopté en fait comme mesure, comme étalon, un paquet de monnaies, mais des paquets de monnaies qui diffèrent d'un pays à l'autre. L'écu, c'est un paquet de monnaies identique vis-à-vis duquel on mesurera les modifications de taux de change de chacun des pays participants.

Ceci est infiniment plus logique que de mesurer les monnaies en dollars, puisque les relations commerciales et financières de chaque pays européen avec les autres pays de la communauté sont en fait quatre à vingt fois supérieures au volume de leurs exportations vers les Etats-Unis. En outre, il y a toute une série d'autres pays, pas seulement les pays de la Communauté actuelle, mais les pays en voie d'adhésion comme l'Espagne et d'autres pays comme la Suisse, la Suède, l'Autriche, etc. qui, inévitablement - et ils l'ont dit déjà - graviteront également vers le système monétaire européen. Et ce qui est vrai pour les autres pays européens de l'OCDE est également vrai de la plupart des pays du Moyen-Orient, des pays d'Afrique, des pays du COMECON pour qui le commerce avec l'Europe est beaucoup plus important que leur commerce avec les Etats-Unis.

L'écu constitue donc un taux de référence, un centre de gravité logique pour la définition de la stabilité éventuelle des taux de change et pour leurs réajustements dans la période initiale.

Secondo, l'écu est déjà utilisé maintenant pour les règlement officiels intracommunautaires entre banques centrales. Chaque banque centrale a maintenant un comptechèque, si vous voulez, en écus auprès du FECOM, du Fonds Européen de Coopération Monétaire.

Troisièmement, et c'est cela ce dont nous aurons sans doute à parler dans notre discussion, ceci devrait entraîner, à plus ou moins brève échéance, l'utilisation beaucoup 
plus large de l'écu, non seulement par les secteurs officiels, mais également par les secteurs privés.

2) Le second trait essentiel du système monétaire européen, c'est d'essayer d'éviter des fluctuations non indispensables des cours de change, et pour cela on a constitué, vous le savez, un Fonds Européen de Coopération Monétaire qui se transformera bientôt en Fonds Monétaire Européen, un Fonds qui assemble une masse de manœuvre de plus de 30 milliards de dollars. Chaque pays a déjà versé $20 \%$ de ses réserves d'or et de dollars à ce Fonds, contre un compte en écus. Ce n'est qu'un premier pas. Si le système monétaire européen se développe comme nous l'espérons vers une union monétaire européenne, ces $20 \%$ deviendront $40 \%, 60 \%, 80 \%$, etc., jusqu'au moment où on aboutira à la création d'un véritable système fédéral de réserves de l'Europe.

Dans l'immédiat, cependant, il importe d'accélérer les réajustements, soit de politique monétaire interne, soit de taux de change, qui sont indispensables à la stabilisation ultime éventuelle des taux de change. Et à cet égard, le système monétaire européen introduit une innovation absolument spectaculaire par rapport à Bretton Woods et par rapport au système du serpent, l'indicateur de divergence.

3) Je ne vais pas m'étendre trop sur cet aspect technique, mais dire simplement qu'autrefois, le seul pays qui pouvait ouvrir une discussion sur une modification de taux de change était le pays qui souhaitait faire changer le taux de change pour sa propre monnaie. Mais tous les pays du monde pouvaient trouver que le mark allemand était trop bas, ou le franc français ou la lire trop hautes, personne ne pouvait en parler sauf l'Allemagne, la France, l'Italie. Maintenant, au contraire, lorsque la monnaie d'un pays s'écarte trop de la moyenne par rapport à l'écu, c'est ce pays sur lequel repose la présomption de devoir modifier sa politique interne ou s'il ne peut pas le faire, son taux de change. Il y a là un facteur nouveau, absolument fondamental dans le désir d'accélérer les réajustements indispensables pour permettre finalement la stabilisation des monnaies européennes. Et pour ceux que cela intéresserait, je leur demande de lire le volume sur le SME, le système monétaire européen, que nous avons publié à Louvain-la-Neuve dans des conditions absolument innovatrices également. Nous avons tenu une conférence internationale sur le SME dix jours après que le système monétaire européen ait été mis en place, les 24 et 25 mars 1979, et nous n'avons pas dû attendre six mois ou six ans pour que ses débats paraissent en texte imprimé, mais, grâce à la collaboration de la Banque de Belgique, le compte-rendu total des discussions de cette première conférence internationale sur le SME a été publié dans les deux mois. J'ai également innové à cet égard, parce que chaque fois que nous discutons des problèmes soulevés par le système monétaire européen, chacun veut toujours renvoyer la balle à l'autre. Si je parle à des privés, ils me disent : " mais il faut d'abord que les officiels fassent ceci ou cela »; si je parle aux officiels, ils ne peuvent rien faire jusqu'à ce que le marché privé ait avancé ; si je parle aux banquiers, ils me disent : « ce n'est pas nous, ce sont nos clients qui nous disent ce que nous devons faire». Chacun renvoie la balle à l'autre. Alors je dis : pour que le système se développe, il faut que chacun fasse ce que lui-même peut faire au lieu de toujours regarder ce que l'autre devrait faire. J'ai donc mis notre volume en vente à un prix exprimé en écus, payables évidemment en monnaie nationale, au cours du change du jour, que vous trouverez tous les jours dans la presse ou sur votre petit écran Reuters. 
4) Enfin, quatrième point essentiel du système monétaire européen, c'est qu'il crée une méthode opérationnelle, une méthode pratique de mise en œuvre du principe affirmé dans le Traité de Rome que les questions de taux de change sont des questions d'intérêt commun. Cette pieuse résolution est maintenant devenue opérationnellement inévitable, parce que comme chaque monnaie définit sa valeur par rapport à l'écu, qui est une moyenne, il est devenu impossible de changer la parité d'une des monnaies du système sans changer la valeur des autres monnaies, et ceci est une décision concertée.

\section{III}

J'en viens aux perspectives d'avenir. J'en soulignerai très rapidement quatre.

1) La première, c'est l'insertion de ce système monétaire européen dans un système mondial. Quelles sont les relations qui s'établiront entre l'écu et les autres monnaies, le franc suisse par exemple, et évidemment le dollar ? Je crois que, s'il n'y a pas une coopération intense entre les Etats-Unis et les autorités européennes, la situation pourrait être tragique et que la création de l'écu pourrait provoquer un affaiblissement plus prononcé du dollar, beaucoup étant tentés de sortir du dollar pour prendre des écus ou des monnaies européennes renforcées par le SME.

Heureusement je crois que cela est une hypothèse absolument invraisemblable parce qu'il est de l'intérêt le plus vital, tant des pays européens que des Etats-Unis, d'éviter une dégradation continue du dollar, qui ne manquerait pas de provoquer des réactions protectionnistes en Europe contre ce que l'on appellerait un dumping de change des Etats-Unis, et de provoquer peut-être même également une panique aux Etats-Unis, voire le contrôle des changes.

En ce qui concerne l'aspect concurrentiel des taux de change actuels, les économistes discutent à perte de vue, à savoir quel devrait être le taux concurrentiel exact du dollar par rapport aux monnaies européennes. J'éviterai d'entrer dans tous ces détails, mais je citerai simplement une expérience personnelle que vous retiendrez tous.

Pendant vingt ans, j'attendais de venir en Europe pour acheter un costume à moitié prix de ce que j'aurais payé à New Haven. Aujourd'hui, j'attends de rentrer à New Haven pour acheter un costume à moitié prix de ce que je paierais à Bruxelles. Il n'y a pas de doute que le dollar est déjà extrêmement sous-évalué aujourd'hui et ne peut pas se sous-évaluer davantage sans rendre impossible la situation de diverses industries européennes.

Donc, il faut que les Etats-Unis et l'Europe coopèrent à défendre cet aménagement concerté des cours du dollar. Ceci requiert évidemment que les Etats-Unis s'engagent dans les réformes fondamentales indispensables pour réajuster les déficits énormes, insoutenables, de leur balance des paiements. Le pays le plus riche du monde ne devrait pas avoir un déficit en compte-courant de 15 milliards de dollars par an ; il devrait, d'après les déclarations répétées que nous faisons nous-mêmes aux NationsUnies, avoir un excédent d'au moins $1 \%$ de son revenu national, ce qui voudrait dire 20 milliards de dollars. Nous en sommes loin. Mais il faut évidemment travailler dans cette direction. Il faudra pour cela mettre en place aux Etats-Unis une véritable politique énergétique et une politique anti-inflationniste plus efficace qu'elle ne l'a été jusqu'ici.

Mais à mettre les choses au mieux, tout cela prendra encore du temps. Même avec la meilleure politique, les meilleurs chances de succès, ce n'est pas du jour au 
lendemain que l'on modifie aussi radicalement une situation économique. Il faut s'attendre à ce que pendant encore deux ou trois ans, la balance des paiements des EtatsUnis reste déficitaire. Pendant cette période de déficits décroissants mais continus, un financement intérimaire devra être assuré, en partie tout au moins, par les pays créanciers. Je crois que ce financement a été rendu possible par le renversement total et vraiment fondamental de la politique américaine annoncée et inaugurée le $1^{\text {er }}$ novembre 1978.

Il y a quatre points dans ce renversement total de politique, contraire à tout ce que l'on avait déclaré jusque-là. Primo, c'est que les Etats-Unis acceptent maintenant lobligation d'interventions massives pour empêcher des fluctuations excessives du dollar; secondo, que les Etats-Unis acceptent de ne plus emprunter uniquement aux banques centrales, mais d'emprunter au marché, aux secteurs privés ; troisièmement, pour permettre cela ils acceptent d'emprunter non seulement en dollars, mais dans la monnaie des pays créanciers : franc suisse, yen, mark allemand. J'ajoute à cela un quatrième point, qui a convaincu, je pense, pas mal de mes amis dans l'administration américaine et surtout au congrès américain. C'est que nous pourrions également considérer la possibilité d'emprunter en écus dès que les emprunts en écus deviendront possibles. Des emprunts en écus seraient moins onéreux en ce sens que la garantie de change serait exprimée en une moyenne de diverses monnaies plutôt que dans une monnaie seule. Donc, il y aurait moins de risques de change. Mais avant tout - et c'est surtout cela qui a impressionné mes amis sur le plan politique, c'est que, au lieu de faire apparaître le dollar comme un satellite du mark, ce serait une démonstration spectaculaire de la volonté réaffirmée de coopération des Etats-Unis à la construction du système monétaire européen. Et il y a pas mal d'indications que les Etats-Unis seraient heureux de s'engager dans cette voie.

Je voudrais ajouter que le nouveau système monétaire européen, le FECOM aujourd'hui, le Fonds Monétaire Européen demain, devrait permettre de donner à cet égard des possibilités que nous n'avions pas auparavant, et notamment la possibilité de consolider ces excédents de dollars, le "overhang» de dollars, dans le cadre du FECOM tout en conservant pour les banques centrales qui sont membres du FECOM la liquidité entière de leurs avoirs.

Je crois que ces avoirs en dollars pourraient être transformés dans la forme d'emprunts la plus prestigieuse du $\mathrm{XIX}^{\mathrm{e}}$ siècle, c'est-à-dire en rentes perpétuelles " consols » en anglais - , c'est-à-dire une obligation donnant une garantie de change et des intérêts acceptables, mais qui n'imposerait pas une date de remboursement précise. Le débiteur remboursera lorsqu'il le pourra, soit en rachetant ses obligations au marché, soit sous une autre forme, mais il ne pourra en fait rembourser que s'il devient excédentaire.

C'est l'une des choses que vous diront tous les économistes, c'est qu'en fait il n'est pas possible de rembourser réellement la dette d'un pays, sauf en accumulant des excédents. Il n'est pas possible non plus pour un pays d'être remboursé sauf en accumulant des déficits. Malheureusement, c'est là une vérité fondamentale que la plupart des gens, et même des officiels, hélas ignorent : à cause de l'habillage financier que l'on donne à ces opérations et qui semblent permettre un remboursement alors qu'elles ne font que redistribuer entre créanciers et emprunteurs la charge des emprunts. 
Un tel système ajusterait enfin l'habillage financier à la réalité des choses au lieu de la dissimuler.

2) Second point, le système monétaire européen ne réussira que si les secteurs privés y croient eux-mêmes et aident à son succès. Je veux souligner ici que presque toutes les grandes réformes monétaires nationales ou internationales ont été faites par le secteur privé et non par le secteur officiel.

Quelle est, pour ne citer qu'un exemple, la grande réforme monétaire des derniers siècles, heureuse ou malheureuse, d'ailleurs? C'est le remplacement des monnaies d'or et d'argent par la monnaie de papier, billets de banque ou dépôts en banque. Cela a été fait au cours du XIX $\mathrm{XI}^{\mathrm{e}}$ siècle lentement, graduellement, par le secteur privé, par le système bancaire. Dès 1913, $85 \%$ des paiements se faisaient déjà par des comptes de chèque et des billets de banque, mais les gouvernements ne s'en rendaient même pas compte.

Je me rappelle que, lorsque je suis rentré en Europe comme représentant du Fonds Monétaire en 1947 et 1948, j'ai dû discuter pendant des semaines avec la Banque de France et la Banque d'Italie pour faire admettre que l'on compte les dépôts de chèque comme partie de la masse monétaire. On n'y croyait pas encore, même en 1947.

Le secteur privé a donc un grand rôle à jouer à cet égard, et la Ligue européenne de coopération économique m'avait demandé en février 1978 ce que les banques pourraient faire pour aider au développement du système monétaire européen. C'était à un moment où, en dépit de l'initiative de Monsieur Jenkins, tout le monde était extrêmement pessimiste quant aux possibilités d'action sur le plan communautaire, sur le plan officiel. Je me suis donc contenté d'une proposition extrêmement modeste, mais qui a été adoptée à l'unanimité par la Ligue européenne qui représente un grand nombre d'importantes affaires européennes. Ma proposition était très simple : les banques devraient, dans l'intérêt de leurs clients, leur permettre d'emprunter ou de déposer en écus aussi bien qu'en monnaies étrangères. Je veux bien que les gouvernements objectent à voir leur monnaie nationale remplacée par l'écu, mais c'est un fait qu'aujourd'hui, d'après les dernières estimations de la Banque des Règlements Internationaux, les banques européennes ont des actifs et des passifs se montant à plus de 600 milliards de dollars à l'étranger, et dans ces opérations avec les étrangers, il y a un peu plus d'une centaine de milliards qui sont dans la monnaie nationale de la banque intéressée et il y en a 500 milliards, plus de $80 \%$, qui sont dans une monnaie étrangère ; eurodollar surtout, euromark, euro franc suisse, etc. L'écu devrait prendre sa place sur ce marché. Je ne dis pas qu'il doive l'absorber, mais il devrait y prendre sa place et c'est un marché de 500 milliards de dollars. Certaines banques l'ont compris et notamment plusieurs banques belges ont commencé à ouvrir des comptes de dépôts et des comptes d'emprunts en écu à leurs clients. J'admets que le client qui désire déposer préférerait peut-être la monnaie qu'il juge devoir être la plus forte, mais le client qui veut emprunter ne désire pas emprunter dans la monnaie la plus forte, il désire emprunter dans la monnaie la plus faible. La seule façon de faire des affaires, c'est de couper la poire en deux et d'emprunter dans une monnaie qui reflète les fluctuations moyennes de l'ensemble des monnaies européennes.

3) Le troisième pas, mais plus lointain évidemment, interviendra le jour où l'on aura réussi suffisamment dans les politiques de stabilisation et les politiques monétaires 
internes pour reprendre une nouvelle initiative concernant l'Union Monétaire Européenne. A cet égard, l'existence de l'écu facilitera grandement la période de transition.

Dans le plan Werner, on ne prévoyait que des monnaies nationales pendant dix ans, pendant toute la période de transition. Et à la fin de la période de transition, lorsque l'on aurait eu une période suffisante de stabilité entre les monnaies nationales, soudainement on jetterait toutes les monnaies nationales par la fenêtre et on adopterait une nouvelle monnaie européenne, inexistante jusque-là. C'était un peu abrupt et difficile à concevoir.

Au contraire, si l'écu existe déjà, a pris sa place dans toutes les transactions internationales, il suffirait alors à chaque pays de permettre à la lumière de sa propre expérience, d'élargir progressivement le champ légal d'utilisation de l'écu à diverses transactions internes, jusqu'à ce que finalement l'écu remplace les monnaies nationales. Mais ceci c'est encore lointain, c'est quelque chose sur lequel les gaullistes ne seraient pas encore d'accord aujourd'hui. Mais même le gaulliste le plus acharné devrait être enchanté, je pense, de voir l'écu se substituer dans l'immédiat, non pas au franc français, mais au dollar et au mark allemand.

4) Finalement et à plus long terme encore (non pas à très long terme, mais peut-être à moyen terme, dans un an ou deux) le succès du système monétaire européen devrait permettre de reprendre sérieusement les efforts de réforme monétaire dans le cadre mondial.

Ce qui est essentiel, c'est quelque chose que l'on puisse négocier avec les EtatsUnis, dans lequel les Etats-Unis puissent prendre leur place. Les Etats-Unis doivent coopérer avec l'Europe à la création du système monétaire européen. Cela nous indiquera dans quelle voie on peut aller pour réformer le Fonds Monétaire International lui-même et en faire, je l'espère, un Fonds monétaire beaucoup plus décentralisé que par le passé, un Fonds monétaire où toutes les décisions ne doivent pas être prises à Washington, mais où beaucoup de décisions affectant par exemple les relations entre la France et la Belgique peuvent se prendre à Bruxelles, dans le cadre du système monétaire européen; où les relations entre la Bulgarie et la Roumanie peuvent se prendre dans le COMECON ; un Fonds qui permettrait de donner une autorité beaucoup plus grande aux groupes régionaux et qui ne réserverait au Fonds Monétaire Mondial que les problèmes dépassant le cadre régional, ceux qui se posent entre groupes régionaux.

Je crois que ce serait infiniment plus efficace pour le fonctionnement du Fonds lui-même et que ça soulèverait moins d'objections économiques, financières et politiques de la part de certains pays, comme les pays sous-développés par exemple, et les pays de l'Est.

\section{IV}

Mais il est temps que je conclue. Je le ferai en répondant d'abord à l'objection qui est certainement sur toutes vos lèvres, n'est-ce pas : "Tout cela est très beau, mais très utopique ; la politique c'est "l'art du possible"; je suis leur chef, donc je dois les suivre», comme le dit un vieux proverbe français.

«Ecoutons le dernier sondage d'opinion pour savoir quel est notre avis ». C'est là la politique des petits politiciens. Mais il y a deux grands hommes d'Etat, aussi 
différents l'un de l'autre que le cynique Talleyrand et l'idéaliste Jean Monnet, mais tous deux grands réalisateurs en Europe, qui avaient une toute autre vue de ce qu'était l'art de la politique. Talleyrand disait que c'était l'art de percevoir à temps l'inévitable et de le préparer dans l'ordre. Jean Monnet disait que "c'est de rendre possible demain ce qui semble encore impossible aujourd'hui ». C'est ce à quoi je vous invite tous à collaborer. 